\title{
Moyamoya syndrome occurred in a girl with an inactive systemic lupus erythematosus
}

\author{
Yun-Jin Lee, MD, Gyu Min Yeon, MD, Sang Ook Nam, MD, PhD, Su Yung Kim, MD, PhD \\ Department of Pediatrics, Pusan National University Children's Hospital, Pusan National University School of Medicine, Yangsan, Korea
}

We report the case of a 17-year-old Korean girl with systemic lupus erythematosus (SLE) who presented with sudden weakness of the right-sided extremities and dysarthria. Oral prednisolone was being taken to control SLE. Results of clinical and laboratory examinations did not show any evidence of antiphospholipid syndrome or thromboembolic disease nor SLE activity. Cerebral angiography showed stenosis of the left internal carotid artery and right anterior cerebral artery with accompanying collateral circulation (moyamoya vessels). After the patient underwent bypass surgery on the left side, she recovered from the neurological problems and did not experience any additional ischemic attack during the 14-month follow-up period. This case represents an unusual association between moyamoya syndrome and inactive SLE (inactive for a relatively long interval of 2 years) in a young girl.

Key words: Moyamoya disease, Systemic lupus erythematosus, Child
Corresponding author: Su Yung Kim, MD, PhD Department of Pediatrics, Pusan National University Children's Hospital, Pusan National University School of Medicine, 20 Geumo-ro, Mulgeum-eup, Yangsan 626-770, Korea

Tel: +82-55-360-3151

Fax: +82-55-360-2181

E-mail: suyung@pasan.ac.kr

Received: 6 October 2012

Revised: 3 January 2013

Accepted: 9 May 2013

\section{Introduction}

Moyamoya disease (MMD) is a cerebrovascular disorder of unknown etiology that is recognized by bilateral progressive stenosis or occlusion of the terminal portions of the internal carotid artery (ICA) and the development of an abnormal vascular network or moyamoya vessels (MMVs) around the arterial occlusion ${ }^{1)}$. Moyamoya syndrome (MMS) is a term applied to describe the MMVs associated with underlying causes such as vasculitis, fibromuscular dysphasia, sickle cell disease, or others. Several reports have described the association between MMS and autoimmune diseases ${ }^{2-55}$.

Only a few reports have linked MMS with systemic lupus erythematosus (SLE) (Table $1)^{2,5-8)}$. A Korean girl presented symptoms of severe headache within one year after diagnosis of SLE diagnoses, which was then diagnosed as a familial MMS associated with SLE ${ }^{5}$. Another 18-year-old Korean woman exhibited MMS associated with the aggravated active lupus nephritis within the first week of SLE diagnosis ${ }^{6}$. A 20-year-old Chinese woman showed sudden right hemiplegia, and was disagnosed as bilateral MMS associated with deteriorating SLE, whose symptoms of SLE started at the age of 10 years ${ }^{8)}$.

In this report, we present an unusual case of patient who presented sudden weakness of right-sided extremities and dysarthria without other associated SLE disease activity at 2 years after SLE onset, and who completely recovered without further ischemic attack through bypass surgery. We also present a literature review of the few reported MMS cases associated with SLE.
Copyright (C) 2013 by The Korean Pediatric Society

This is an open-access article distributed under the terms of the Creative Commons Attribution NonCommercial License (http://creativecommons.org/ licenses/by-nc/3.0/) which permits unrestricted noncommercial use, distribution, and reproduction in any medium, provided the original work is properly cited. 


\section{Case report}

A 17-year-old Korean female was brought to the pediatric department because of new-onset, right-sided weakness that was first noted 3 weeks ago when it affected her writing ability. Her speech was slurred and she complained tongue numbness. Her symptoms seemed to improve for a while at first, but afterward she did not completely recover for 1 month. There was no evidence of trauma, flu-like symptoms, or no report of nausea, vomiting, headache, or convulsions. Evaluating the patient's medical history, there were no complications at birth, and her development had been age appropriate. There was no family history of stroke in young individuals.

In September 2008, at 15 years of age, she was diagnosed with lupus nephritis. Initial clinical course showed severe manifestations with microangiopathic hemolytic anemia, thrombocytopenia, and hypertension. The renal biopsy finding indicated the lupus nephritis ISN/RPS Class IV-G(A). After the initial period of 6 months, her clinical symptoms were well controlled with treatment of azathioprine, prednisolone and antihypertensive drugs. However, after 22 months, new-onset right-sided weakness developed.

On admission, vital signs were stable. Neurological examination revealed asymmetric motor weakness with the grade of $4 / 5$ in the right arm and leg. She was mentally alert, but had a subtle dysarthric speech despite of normal cranial nerves. Reflexes for both knees were symmetric and normal. Otherwise, her systemic examination was unremarkable.

The laboratory tests revealed the followings: leukocyte, 5,100/ $/ \mathrm{mm}^{3}$; hemoglobin, $14.8 \mathrm{~g} / \mathrm{dL}$; platelet, $266,000 / \mathrm{mm}^{3}$; erythrocyte sedimentation rate, $2 \mathrm{~mm} / \mathrm{hr}(0-20)$; C-reactive protein level, $0.01 \mathrm{mg} / \mathrm{dL}$ (0-0.5); C3, $64 \mathrm{mg} / \mathrm{dL}$ (79-152); C4,
$12.4 \mathrm{mg} / \mathrm{dL}(10-40)$; and CH50, $37.6 \mathrm{U} / \mathrm{mL}$ (23-46). Electrolytes, renal and hepatic profiles, and lipid profile were normal. Anti-thrombin III, prothrombin time, activated partial thromboplastin time, protein C (120\%), protein S (70\%), and lipid profiles were all within normal limits. Urine analysis showed no proteinuria or microscopic hematuria. Immunologic evaluation revealed antinuclear antibody (ANA) of 1:80, antids-DNA immunoglobulin G (IgG)/IgM 7 (0-25)/14 (0-20) $\mathrm{IU} / \mathrm{mL}$, anticardiolipin IgG/IgM (-/-), lupus anticoagulant (-), antiphospholipid antibody (-), anti-Ro/anti-La (-/-), and venereal disease research laboratory test (VDRL) (-). Chest $\mathrm{X}$-ray, electrocardiogram, and echocardiogram findings were all unremarkable. There was no evidence of aggravation of SLE activity.

Brain magnetic resonance imaging (MRI) revealed no evidence of cerebral infarction or ischemic change (Fig. 1). Magnetic resonance angiography of the brain revealed stenosis of the supraclinoid portion of the left ICA and the right anterior cerebral artery (ACA). Conventional angiography showed severe narrowing of the distal portion of left ICA, the proximal portion of left middle cerebral artery (MCA), ACA and the right ACA (Fig. 2). These findings were compatible with MMD.

The patient was started aspirin, however, her right-sided weakness and dysarthria continued unabated. One week later, left encephalo-duro-arterio-synangiosis bypass surgery was performed. Postoperative MRI showed no interval change of right MCA branch and no further progression with new lesion. She has since recovered without new ischemic symptoms. No further neurological deficits occurred during a 14-month followup. Right-side surgery will be scheduled depending on follow-up neuroimaging results and the patient's neurological symptoms.

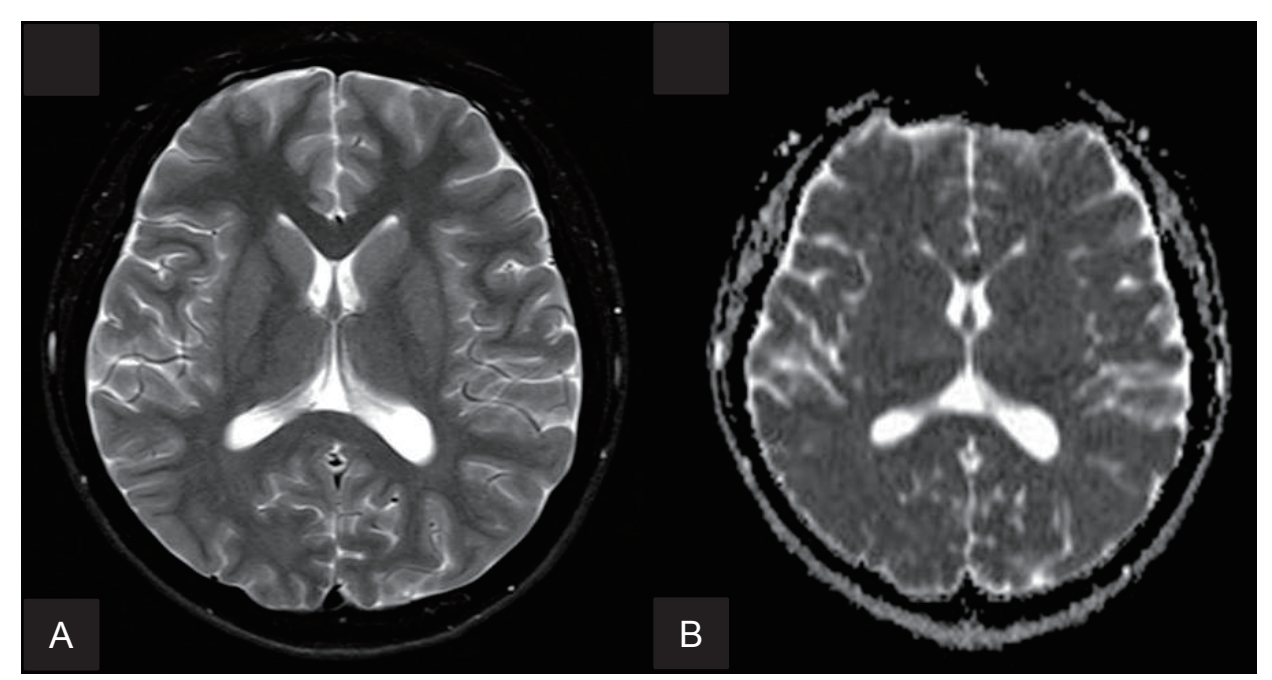

Fig. 1. Brain magnetic resonance imaging (MRI). Findings of axial T2-weighted MRI (A) and diffusion-weighted MRI (B) at the basal ganglia level are unremarkable, with no evidence of acute infarctions. 

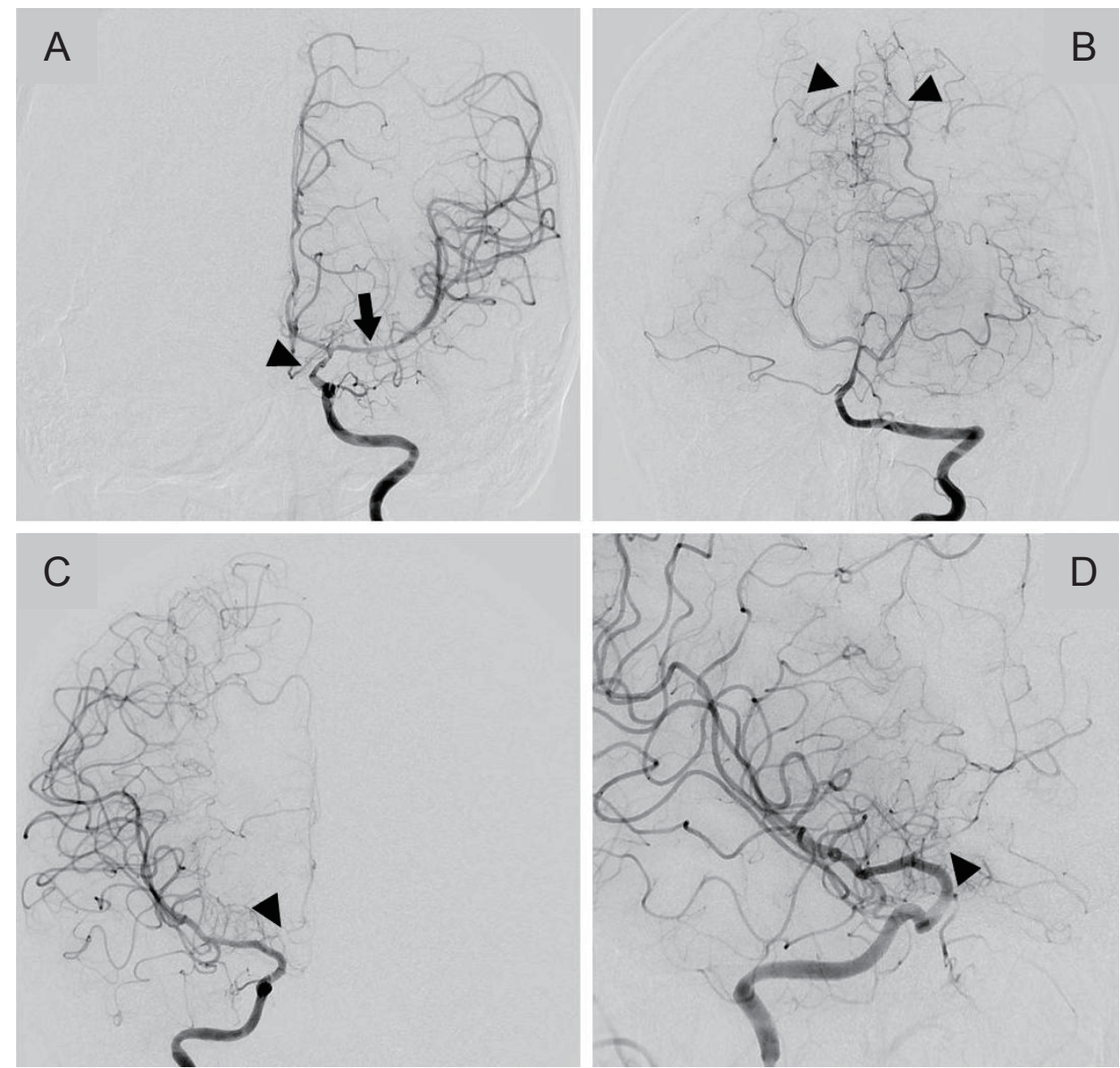

Fig. 2. BConventional cerebral angiograms of both carotid arteries. (A) Left internal carotid arteriogram shows diffuse stenosis of the supraclinoid portion of the left internal carotid artery (arrowhead) and the proximal portion of the left middle cerebral artery (arrow) with (B) collateral circulation from the left posterior cerebral artery (arrowheads). (C, D) Right internal carotid arteriogram shows severe stenosis of the A1 portion of the right anterior cerebral artery (arrowheads).

\section{Discussion}

Our patient was a remarkable case of MMS in a 17-year-old girl with SLE. The right-side hemiparesis developed at 2 years after SLE onset without SLE disease activity. The incidence of cerebrovascular accidents (CVA) in SLE cases is 2\% to 12\% within the first several years ${ }^{9,10)}$. Symptomatic occlusion or stenosis of large cerebral vessels in SLE usually occurs within several years of SLE onset during the active period of the illness. However, cerebrovasculopathy in SLE may occur even without vigorous disease activity ${ }^{11)}$.

There have been few reported MMS cases associated with SLE (Table 1$)^{2,5-8)}$. Matsuki et al. ${ }^{7)}$, reported the first case of SLE and Sjögren syndrome associated with unilateral MMVs in cerebral arteries in the absence of antiphospholipid syndrome. The patient was a 30-year-old Japanese woman who presented with recurrent, right-sided homonymous hemianopsia without major organ involvement of SLE.
An 18-year-old Saudi male with inactive SLE presented right-sided hemiparesis one year after SLE onset without any accompanying risk factors for $\mathrm{CVA}^{2)}$. Angiography revealed bilateral ICA stenosis with MMVs. This patient was the first documented case of SLE associated "bilateral" MMVs. He was medically treated for 3 weeks before the decision to perform bypass surgery. Despite the surgery, he developed new ischemic stroke and multiple cranial nerve palsies. Reoperation and medical treatment was performed, and no additional attacks occurred during a 9-month follow-up period.

Lee et $\mathrm{al}^{5}{ }^{5}$, described a 13-year-old Korean girl with SLE who developed severe migraine-type headaches one year after onset. Patient's mother had been diagnosed with MMD. There was no symptomatic evidence of SLE disease activity or other risk factors of CVA. However, her magnetic resonance angiography revealed bilateral ICA stenosis with collateral circulation. Headache can be important indicator of MMD in SLE, occurring in a significant numbers of patients ${ }^{12)}$. 
Table 1. Previous reports on MMS associated with SLE

\begin{tabular}{|c|c|c|c|c|c|c|}
\hline Author & Matsuki et al." & El Ramahi and Al Rayes ${ }^{2)}$ & Lee et al..$^{5}$ & Jeong et al. ${ }^{6}$ & Mok and Poon ${ }^{8)}$ & This patient \\
\hline Country & Japan & Saudi & Korea & Korea & China & Korea \\
\hline $\begin{array}{l}\text { Sex/age at onset } \\
\text { of MMS }\end{array}$ & Female/30 yr & Male/18 yr & Female/13 yr & Female/18 yr & Female/20 yr & Female/17 yr \\
\hline $\begin{array}{l}\text { Interval between } \\
\text { MMS and SLE }\end{array}$ & $1 \mathrm{yr}$ & $1 \mathrm{yr}$ & $1 \mathrm{yr}$ & Within 1 wk & $10 \mathrm{yr}$ & $2 \mathrm{yr}$ \\
\hline $\begin{array}{l}\text { Symptoms at } \\
\text { onset of MMS }\end{array}$ & $\begin{array}{l}\text { Right-sided homonymous } \\
\text { hemianopsia }\end{array}$ & $\begin{array}{l}\text { Right-sided hemiparesis, } \\
\text { dysarthria }\end{array}$ & $\begin{array}{l}\text { Intractable } \\
\text { headache }\end{array}$ & Left-sided hemiparesis & $\begin{array}{l}\text { Right-sided } \\
\text { hemiplegia }\end{array}$ & $\begin{array}{l}\text { Right-sided hemiparesis, } \\
\text { dysarthria }\end{array}$ \\
\hline SLE activity & Inactive & Inactive & Inactive & Active & Active & Inactive \\
\hline $\begin{array}{l}\text { Other medical } \\
\text { history }\end{array}$ & Sjögren syndrome & & Maternal MMD & Nephritis, dyslipidemia & & \\
\hline ICA involvement & Unilateral (left) & Bilateral & Bilateral & Bilateral & Bilateral & Bilateral \\
\hline Treatment & $\begin{array}{l}\text { Medication \& bypass } \\
\text { surgery }\end{array}$ & Medication \& bypass surgery & $\begin{array}{l}\text { Supportive } \\
\text { medication }\end{array}$ & $\begin{array}{l}\text { Medication \& bypass } \\
\text { surgery }\end{array}$ & $\begin{array}{l}\text { Medication \& } \\
\text { rehabilitation }\end{array}$ & $\begin{array}{l}\text { Medication \& bypass } \\
\text { surgery }\end{array}$ \\
\hline Outcomes & Reduction (1-2/mo) & $\begin{array}{l}\text { New ischemic stroke, } \\
\text { hemiplegia, global gphasia }\end{array}$ & Mild headache & Good & Partially improved & Good \\
\hline
\end{tabular}

MMS, Moyamoya syndrome; SLE, systemic lupus erythematosus; ICA, internal carotid artery.

Another 18-year-old Korean woman had sudden onset of left hemiparesis while being treated for active lupus nephritis within the first week of SLE diagnosis ${ }^{6}$. She did not have any history of seizure, or cardiovascular disorder. Brain MRI and angiography revealed multiple cerebral infarctions, and bilateral stenosis of the ICA was observed with abundant MMVs, which were consistent with MMS diagnosis.

A 20-year-old Chinese woman with SLE had sudden right hemiplegia, whose illness started at the age of 10 years ${ }^{8)}$. She showed some evidence of active SLE as lupus flare with arthritis, proteinuria and deteriorating serology. Conventional angiography revealed bilateral occlusion of the supraophthalmic segments of the ICAs with extensive collateral MMVs. She was treated with pulse methylprednisolone, anticoagulation and rehabilitation for 3 months, and is scheduled for pial synangiosis. Among the previous reported five cases ${ }^{2,5-8)}$, this Chinese woman showed the longest duration of the interval between initial SLE onset and subsequent diagnosis of MMS, which ranged from 1 week to 10 years.

The mechanism for manifestation of MMS following autoimmune disease remains unclear. However, MMS with positive indicators for ANA, anti-ds-DNA, anti-Ro, or lupus anticoagulant, may suggest the immunological role in MMS pathogenesis ${ }^{13)}$. Jeong et al. ${ }^{6}$, hypothesized that underlying cerebrovascular lesions, such as MMVs with coexisting high lupus activity, would become more susceptible to CVA. In addition, hemodynamic instability due to long-term use of diuretic and antihypertensive drugs might stimulate ischemic change associated with MMVs. Our patient was negative in anticardiolipin antibodies and VDRL, and had no clinical features indicating antiphospholipid syndrome. She also had no evidence of thromboembolic disease. Her angiographic findings showed typical of MMD and we could speculate a related MMS following her SLE, even though no SLE disease activity was detected for 2 years. Also, we suggest a hidden genetic factor may play a role in her MMS, although she has no reported family history of MMD.

Of the six patients diagnosed with MMS associated with SLE including our case, all were Asian including 3 Koreans, one Japanese, one Saudi Arabian and one Chinese, and five were female ${ }^{2,5-8)}$. The known strong prevalence of MMD in Asian, especially in Japan, suggests that a genetic trait associated with the disease ${ }^{14)}$. MMD occurs also more frequently in female, with a female to male ratio of 1.8 to $2.18: 1$, and peak incidences are at aged 1-10 and 30-50. The bimodal age distributions as well as genetic trait of MMD may affect a diverse interval between initial SLE and following MMS.

In conclusion, we present an unusual case of MMS in a girl with SLE who was successfully treated through the bypass surgery. MMD is observed more frequently in Asia. Therefore, whenever an SLE patient presents symptoms of CVA either with or without disease activity, the related or accidental MMD should be included in the differential diagnosis, particularly in prevalent areas. Additionally, we would like to make an overture that an initial cerebrovascular check up might be helpful to patients of the SLE in order to discover an unusual association of the MMD with the SLE thereafter.

\section{Conflict of interest}

No potential conflict of interest relevant to this article was reported. 


\section{Acknowledgments}

This study was supported by a 2012 research grant from Pusan National University Yangsan Hospital.

\section{References}

1. Suzuki J, Takaku A. Cerebrovascular "moyamoya" disease. Disease showing abnormal net-like vessels in base of brain. Arch Neurol 1969;20:288-99.

2. El Ramahi KM, Al Rayes HM. Systemic lupus erythematosus associated with moyamoya syndrome. Lupus 2000;9:632-6.

3. Im SH, Oh CW, Kwon OK, Kim JE, Han DH. Moyamoya disease associated with Graves disease: special considerations regarding clinical significance and management. J Neurosurg 2005;102:1013-7.

4. Joo SP, Kim TS, Lee JH, Lee JK, Kim JH, Kim SH, et al. Moyamoya disease associated with Behcet's disease. J Clin Neurosci 2006;13: 364-7.

5. Lee CM, Lee SY, Ryu SH, Lee SW, Park KW, Chung WT. Systemic lupus erythematosus associated with familial moyamoya disease. Korean J Intern Med 2003;18:244-7.

6. Jeong HC, Kim YJ, Yoon W, Joo SP, Lee SS, Park YW. Moyamoya syndrome associated with systemic lupus erythematosus. Lupus
2008;17:679-82.

7. Matsuki Y, Kawakami M, Ishizuka T, Kawaguchi Y, Hidaka T, Suzuki K, et al. SLE and Sjögren's syndrome associated with unilateral moyamoya vessels in cerebral arteries. Scand J Rheumatol 1997;26:392-4.

8. Mok CC, Poon WL. Moyamoya phenomenon in systemic lupus erythematosus. Rheumatology (Oxford) 2011;50:1931.

9. Sibbitt WL Jr, Brandt JR, Johnson CR, Maldonado ME, Patel SR, Ford CC, et al. The incidence and prevalence of neuropsychiatric syndromes in pediatric onset systemic lupus erythematosus. J Rheumatol 2002;29:1536-42.

10. Brey RL, Holliday SL, Saklad AR, Navarrete MG, HermosilloRomo D, Stallworth CL, et al. Neuropsychiatric syndromes in lupus: prevalence using standardized definitions. Neurology 2002;58:1214-20.

11. Mitsias P, Levine SR. Large cerebral vessel occlusive disease in systemic lupus erythematosus. Neurology 1994;44(3 Pt 1):385-93.

12. Sfikakis PP, Mitsikostas DD, Manoussakis MN, Foukaneli D, Moutsopoulos HM. Headache in systemic lupus erythematosus: a controlled study. Br J Rheumatol 1998;37:300-3.

13. Bonduel M, Hepner M, Sciuccati G, Torres AF, Tenembaum S. Prothrombotic disorders in children with moyamoya syndrome. Stroke 2001;32:1786-92.

14. Burke GM, Burke AM, Sherma AK, Hurley MC, Batjer HH, Bendok BR. Moyamoya disease: a summary. Neurosurg Focus 2009;26:E11. 